\section{Estado nutricional e tempo de tela de escolares da Rede Pública de Ensino Fundamental de Niterói, Rio de Janeiro, Brasil}

\author{
Nutritional status and screen time among public \\ school students in Niterói, Rio de Janeiro State, \\ Brazil
}

Estado nutricional y tiempo de pantalla de escolares de la red pública de enseñanza fundamental de Niterói, Río de Janeiro, Brasil
Marcelo Barros de Vasconcellos 1,2 Luiz Antonio dos Anjos 1,2 Mauricio Teixeira Leite de Vasconcellos 3

\begin{abstract}
1 Programa de Pós-graduação em Saúde Coletiva,

Universidade Federal

Fluminense, Niterói, Brasil.

2 Departamento de Nutrição Social, Universidade Federal Fluminense, Niterói, Brasil. ${ }^{3}$ Escola Nacional de Ciências Estatísticas, Instituto Brasileiro de Geografia e Estatística, Rio de Janeiro, Brasil.

Correspondência

L. A. Anjos

Laboratório de Avaliação

Nutricional e Funcional.

Departamento de Nutrição

Social, Universidade Federal Fluminense.

Rua Mário Santos Braga 30 , Niterói, RJ 24020-140, Brasil. anjos@ensp.fiocruz.br
\end{abstract}

\begin{abstract}
The aim of this study was to assess nutritional status, sedentary behavior (TV, computer, and videogame time and screen time as the sum of these first three) and physical activity using a questionnaire with youth (10 to 18 years of age) enrolled in public schools in Niterói, Rio de Janeiro State, Brazil. Anthropometry (body mass and stature), sedentary behavior, and information on physical activity were obtained in a probability sample of 10 to 18 year-old students ( $n=$ 328; 108 boys) stratified by school and selected in two stages (classes and students). Low height for age did not appear as a problem, but 25.7\% of the youth presented excess weight (18\% overweight and 7.7\% obese). Total screen time did not differ between the sexes, but boys spent more time playing videogames than girls, regardless of age, while girls watched more TV. Boys spent twice as much time as girls of all ages in physical activity (three times more in the $\geq 14$ year-old group). Screen time was significantly associated with excess weight. In conclusion, public school youth in Niterói show high prevalence rates of excess weight associated with inadequate lifestyle.
\end{abstract}

Nutritional Status; Obesity; Adolescent

\section{Resumo}

O presente estudo avaliou o estado nutricional relacionado ao tempo de tela (computado como o somatório de assistir à televisão, usar computador e jogar videogame) e à prática de atividade física, por meio de questionário semiestruturado, em uma amostra probabilística $(n=328$, sendo 108 meninos) de escolares (10 a 18 anos de idade) do sexto ao nono ano da Rede Pública de Ensino Fundamental de Niterói, Rio de Janeiro, Brasil. Déficit de estatura não foi um problema nos escolares, mas 25,7\% tinham excesso de peso, sendo $18 \%$ com sobrepeso e $7,7 \%$ com obesidade. A média de tempo semanal de televisão foi de 24,2h, sendo maior entre as meninas. $O$ tempo de tela não foi diferente entre sexo, mas os meninos, independentemente da idade, passavam mais tempo jogando videogame do que as meninas. Os meninos dedicavam o dobro de tempo realizando atividade física em relação às meninas de todas as idades e o triplo na faixa de 14 anos de idade ou mais. O tempo de tela associou-se significativamente com o excesso de peso. Conclui-se que os escolares da Rede Pública de Ensino Fundamental de Niterói apresentam elevada prevalência de excesso de peso associado a estilo de vida inadequado.

Estado Nutricional; Obesidade; Adolescente 


\section{Introdução}

Como observado em adultos, a prevalência de obesidade vem aumentando nos últimos anos também em adolescentes, o que demonstra a necessidade de medidas preventivas nesta fase para evitar suas complicações na vida adulta. De acordo com a mais recente Pesquisa de Orçamentos Familiares (POF), no Brasil, 20,5\% dos adolescentes estão com sobrepeso e 4,9\% são obesos 1 . Apesar da inexistência de dados objetivos nacionais sobre o balanço energético, um possível aumento na ingestão energética associado ao maior tempo que os jovens dedicam a atividades sedentárias nas últimas décadas pode ajudar a explicar a epidemia de obesidade no país 2 .

O comportamento sedentário tem sido associado a efeitos adversos à saúde em pessoas jovens, no entanto a natureza e o contexto do sedentarismo são mal compreendidos 3 . Em passado recente, a medida do tempo assistindo à televisão era o marcador utilizado para caracterizar um estilo de vida sedentário, pelo fato de a TV ser onipresente, com pelo menos um aparelho na maioria das casas de família nos países desenvolvidos 4 . De fato, pesquisa em amostra de 498 estudantes de baixa renda de quatro escolas públicas em Chicago, Estados Unidos, em 2003, verificou que todos os adolescentes tinham televisão em casa, quase $90 \%$ a tinham no quarto e também por assinatura 5 .

A juventude mundial aderiu à televisão como passatempo dominante em momentos diferentes do dia ${ }^{6}$. Com a explosão da popularidade do acesso à internet e como os adolescentes muitas vezes se envolvem em diversas atividades simultaneamente, medidas mais abrangentes, além da simples medição do tempo assistindo à programação televisiva, tornaram-se necessárias para avaliar o comportamento sedentário diário deste grupo 7. Por isso, nos últimos anos, começaram a surgir análises sobre o tempo de tela, realizadas com base no tempo gasto em entretenimento com vários aparelhos, como televisão, computador ou videogame $3,7,8$, para representar o comportamento sedentário ${ }^{9}$, na presunção de que este tempo estaria substituindo aquele destinado à prática de atividades fisicamente mais vigorosas e, desta forma, contribuindo para o aumento da obesidade. A premissa é que, quanto maior for o tempo de tela, menor será a prática de atividade física; consequentemente, maior será o ganho de peso e a prevalência de obesidade na infância 10.

Dessa forma, o tempo de tela é importante para o entendimento do sedentarismo e a saúde dos adolescentes 11. Como dados populacionais sobre o tema e suas consequências para a saúde em segmentos da população brasileira são escassos, o objetivo principal do presente estudo foi avaliar a inter-relação entre o estado nutricional e o tempo de tela em uma amostra probabilística de adolescentes da Rede Pública Municipal de Ensino Fundamental de Niterói, Rio de Janeiro, Brasil.

\section{Materiais e métodos}

Trata-se de um estudo de corte transversal, com amostra probabilística de escolares do sexto ao nono ano do ensino fundamental das escolas públicas de Niterói. O município conta com aproximadamente 479.386 habitantes, 62.507 dos quais são adolescentes (Instituto Brasileiro de Geografia e Estatística. Niterói - RJ. Ensino - matrículas, docentes e rede escolar 2008. http://www.ibge. gov.br, acessado em 09/Mar/2010). No primeiro semestre de 2010, período de realização do estudo, 4.545 alunos participavam do ensino fundamental público, distribuídos entre o sexto e o nono ano, totalizando 176 turmas em 12 escolas.

Foi selecionada uma amostra estratificada por escola em dois estágios: (1) turmas, com probabilidade proporcional ao seu número de alunos; (2) alunos, usando o procedimento de amostragem inversa, descrito originalmente por Haldane ${ }^{12}$. A amostragem inversa é um método de estatística sequencial que consiste em contar quantas unidades precisam ser contadas para atingir o número prefixado de entrevistas realizadas. Foi o método escolhido por ser a única forma de lidar com as não respostas, sem o viés da reponderação, da substituição ou da sobreamostragem.

O tamanho da amostra foi inicialmente fixado em 45 turmas, sendo alocado por escola de forma proporcional à raiz quadrada do número de alunos na instituição (alocação potência 1/2), que resultou em uma amostra de duas a seis turmas por estrato. Estabeleceu-se que, nas turmas menores, o número de alunos a serem entrevistados seria sete; nas demais, oito, de modo a chegar a um tamanho da amostra de alunos igual a 340, o suficiente para estimar, com nível de significância de $5 \%$, proporções com erro máximo de 0,053 .

Em virtude de problemas nas turmas menores, o procedimento contabilizou 12 perdas, resultando em uma amostra de 328 alunos, sendo 108 meninos e 220 meninas. Foram calculados os pesos amostrais, como o inverso das probabilidades de inclusão em cada estágio da amostra, que permitem estimar, para a totalidade de adolescentes escolares do sexto ao nono ano da Rede Pública Municipal de Ensino Fundamen- 
tal de Niterói, as medidas antropométricas e a caracterização de tempo de tela e de atividade física, obtidas para a amostra.

Após a aprovação formal da realização da pesquisa pela Secretaria de Educação do Município de Niterói, procedeu-se ao contato com a direção de cada uma das 12 escolas, a qual, uma vez esclarecida sobre a importância, os objetivos e a metodologia do estudo, autorizou sua realização. Os critérios de exclusão foram não ter a autorização dos pais para participar, o escolar ter completado 19 anos de idade, ser gestante ou apresentar deficiência física.

As medidas de massa corporal e estatura foram obtidas na própria escola por dois avaliadores (professores de educação física), previamente treinados, em uma sala de aula especificamente adaptada para tal fim. A massa corporal foi aferida uma única vez em balança eletrônica (Seca, Birmingham, Reino Unido), com precisão de $0,1 \mathrm{~kg}$, com o sujeito descalço e com o uniforme escolar. A estatura foi medida em estadiômetro portátil (Seca) que foi afixado em uma parede sem rodapé. $\mathrm{O}$ indivíduo foi posicionado no estadiômetro com os braços ao longo do corpo, pés unidos e centralizados no equipamento, cabeça, nádegas e calcanhares encostados na parede e com a cabeça mantida num plano horizontal (linha de Frankfort), seguindo padronização descrita em Lohman et al. 13. A régua do estadiômetro foi então deslocada até a cabeça do voluntário e realizada a leitura após uma expiração normal. Foram feitas duas aferições da estatura, e a média dos valores foi usada nas análises.

De posse dos dados de massa corporal e estatura, foi calculado o índice de massa corporal (IMC: $\mathrm{kg} / \mathrm{m}^{2}$ ), por meio da divisão da massa corporal $(\mathrm{kg})$ pelo quadrado da estatura $\left(\mathrm{m}^{2}\right)$. Os valores das medidas de estatura, IMC e idade foram usados para calcular os valores $\mathrm{Z}$ dos índices estatura para idade (EI) e IMC para idade (IMC-I), assim como para determinar o estado nutricional dos adolescentes de acordo com os procedimentos propostos pela Organização Mundial da Saúde (OMS) 14. Baixa estatura foi estabelecida para valores de Z EI $<-2$. Valores de Z IMC-I foram usados para identificar sobrepeso $(+1<Z \leq+2)$, obesidade $(Z>+2)$ e baixo peso $(Z<-2)$. $O$ conjunto de sobrepeso e obesidade foi considerado como excesso de peso.

Um questionário semiestruturado de atividades diárias, adaptado de Barros \& Nahas 15 , foi respondido pelo escolar na mesma sala de coleta de dados antropométricos. O jovem respondia se possuía, em casa, aparelhos de televisão, televisão por assinatura ou antena parabólica, videogame para utilização no televisor e computador; respondia também se alguém controlava o tempo que ele passava assistindo aos programas de televisão. A seguir, o escolar anotava o tempo gasto em cada dia da semana e de final de semana vendo $\mathrm{TV}$, jogando videogame e usando o computador. O tempo de tela foi considerado como a soma do tempo assistindo à televisão, jogando videogame e usando computador. Para a estimativa da atividade física, o escolar anotava o tempo e a duração de todas as atividades realizadas nos dias da semana e de final de semana. Ao receber o questionário preenchido, o pesquisador o revisou quanto à sua completude.

A análise dos dados incluiu o cálculo de média, erro padrão e intervalo de $95 \%$ de confiança (IC95\%) para as variáveis contínuas e porcentagens e IC95\% para as variáveis categóricas. Todas as análises foram realizadas com pesos amostrais e com os procedimentos surveyfreq, surveylogistic e surveymeans, do SAS versão 9.2 (SAS Inst., Cary, Estados Unidos), que tratam adequadamente desenhos de amostra complexa. O tempo de tela foi estratificado em três faixas (menos de 14h; 14h$27,9 \mathrm{~h}$ e $28 \mathrm{~h} /$ semana ou mais) para avaliação de sua associação com a prevalência de excesso de peso (sobrepeso + obesidade), mediante regressão logística, cujo resultado foi expresso em odds ratio (OR) e seus IC95\%. A significância dos OR foi estabelecida quando o IC95\% não incluíam a unidade. A significância das diferenças entre médias e proporções por sexo e faixa etária (menor de 14 e 14 anos de idade ou mais) foi estabelecida quando não havia interseção entre os IC95\% correspondentes.

$\mathrm{O}$ projeto atendeu aos requisitos que envolvem os aspectos éticos da pesquisa em seres humanos e contemplou a Resolução no 196/96 do Conselho Nacional de Saúde, tendo sido aprovado pelo comitê de ética da Universidade Federal Fluminense (UFF; CEP CMN/HUAP no 177/2010).

\section{Resultados}

A amostra compreendeu 328 escolares (220 meninas) que representam os 4.546 (3.082 meninas) alunos do sexto ao nono ano da Rede Pública Municipal de Ensino Fundamental de Niterói. A idade dos meninos oscilou entre 10,9 e 18,4 anos e a das meninas entre 10,6 e 17,3 anos (Tabela 1). A média dos valores $\mathrm{Z}$ da estatura para a idade e IMC para idade foram bem próximos de zero. A distribuição dos valores $Z$ estatura para idade foi semelhante à do crescimento da população de referência, fazendo com que a prevalência de baixa estatura fosse baixa e não significativamente diferente entre sexo $(1,4 \%$, IC95\%: 0,$77 ; 1,96$ para os meninos e $0,7 \%$, IC95\%: 0,$42 ; 1,02$ para as 
Idade e medidas antropométricas por sexo dos escolares da Rede Pública Municipal de Ensino Fundamental de Niterói, Rio de Janeiro, Brasil, 2010.

\begin{tabular}{lcccccccc}
\hline \multirow{2}{*}{ Variável } & \multicolumn{3}{c}{ Meninos (N=1.464) } & \multicolumn{3}{c}{ Meninas (N = 3.082) } \\
& Média & EP & Mediana & IC95\% & Média & EP & Mediana & IC95\% \\
\hline Idade (anos) & 13,8 & 0,1 & 13,6 & 13,$5 ; 14,0$ & 13,6 & 0,1 & 13,5 & 13,$4 ; 13,8$ \\
Massa corporal (kg) & 51,6 & 1,3 & 49,9 & 49,$0 ; 54,1$ & 51,1 & 0,7 & 49,8 & 49,$6 ; 52,5$ \\
Estatura (cm) * & 160,9 & 1,1 & 162,9 & 158,$6 ; 163,2$ & 157,4 & 0,4 & 158 & 156,$6 ; 158,3$ \\
IMC (kg/m2) & 19,6 & 0,3 & 18,9 & 19,$0 ; 20,3$ & 20,5 & 0,3 & 19,7 & 20,$0 ; 21,0$ \\
EI & 0,1 & 0,1 & 0,1 & $-0,1 ; 0,3$ & 0,1 & 0,06 & 0,1 & $-0,1 ; 0,2$ \\
IMC-I & 0,2 & 0,12 & $-0,02$ & $-0,1 ; 0,3$ & 0,3 & 0,08 & 0,1 & 0,$1 ; 0,4$ \\
\hline
\end{tabular}

El: valores Z do índice estatura para idade; EP: erro-padrão; IC95\%: intervalo de 95\% de confiança; IMC: índice de massa corporal; IMC-I: valores Z do IMC para idade.

* Estimativas de médias significativamente (5\%) diferentes entre meninos e meninas.

meninas), apesar de os meninos apresentarem estatura média significativamente maior do que das meninas.

Constatou-se que 18,0\% (IC95\%: 16,9; 19,1) dos adolescentes tinham sobrepeso e $7,7 \%$ (IC95\%: 6,94; 8,50) apresentaram obesidade o que corresponde a 25,7\% (IC95\%: 24,5; 27,0) de excesso de peso (sobrepeso + obesidade), não havendo diferença entre meninos $(24,5 \%$, IC95\%: $22,3 ; 26,7)$ e meninas $(26,3 \%$, IC95\%: 24,$8 ; 27,89)$. Baixo peso foi maior nos meninos e obesidade maior em meninas de todas as idades (Tabela 2). Isso é causado pela significativa maior prevalência de baixo peso e menor prevalência de obesidade nos meninos com menos de 14 anos de idade em relação às meninas da mesma faixa etária. As prevalências de sobrepeso e obesidade foram maiores na faixa etária inferior a 14 anos independentemente do sexo.

O percentual de adolescentes que possuíam videogame $(52 \%$, IC95\%: 50,6; 53,5) foi menor do que o daqueles que tinham computador $(66,3 \%$, IC95\%: 64,9; 67,7). Meninos possuíam mais videogame $(73,1 \%$, IC95\%: 70,8; 75,4) e computador $(69,2$, IC95\%: 66,$8 ; 71,6)$ do que as meninas (42\%, IC95\%: 40,3; 43,8\% e 64,9\%, IC95\%: 63,2; 66,6 , respectivamente). Constatou-se que $100 \%$ dos escolares tinham televisão em casa, com média de 2,3 (IC95\%: 2,2; 2,4) aparelhos por residência; quase a metade dos escolares $(47,2 \%$, IC95\%: 45,8; 48,7\%) possuía televisão por assinatura. Identificou-se que o tempo de ver televisão é controlado para 36,7\% (IC95\%: 34,9; 37,7) dos escolares, sendo significante a diferença entre meninos $(32,7$, IC95\%: 30,3; 5,1) e meninas $(38 \%$, IC95\%: 36,3; 39,7) e por faixa etária (menor de 14 anos $=39,8 \%$, IC95\%: 37,9; 41,7; 14 anos ou mais
$=31,7 \%$, IC95\%: 29,6; 33,8). O tempo de assistir televisão é, percentualmente, mais controlado para as meninas com idade superior ou igual a 14 anos do que para os meninos da mesma faixa etária.

A média de tempo total semanal assistindo à programação televisiva foi de 24,2h (IC95\%: 22,5; $25,9)$ ou, aproximadamente, $3,5 \mathrm{~h}$ por dia e variou pouco entre os dias da semana ou de final de semana. O tempo semanal vendo televisão foi significativamente superior para as meninas e o tempo jogando videogame foi significativamente superior para os meninos (Tabela 3). Tal comportamento não impactou o tempo de tela, uma vez que não só o tempo que as meninas se dedicavam à televisão foi similar ao tempo que os meninos se dedicavam ao videogame $(\cong 6 \mathrm{~h})$, como também não houve diferenças entre os sexos quanto à exposição ao computador. Os meninos, independentemente da idade, passavam mais tempo jogando videogame do que as meninas; além disso, em relação à realização de atividade física, eles dedicavam o dobro de tempo, em comparação com as meninas de todas as idades, e o triplo, comparados com as de 14 anos ou mais.

O tempo total semanal assistindo à televisão, jogando videogame ou realizando atividade física não apresentou correlação com nenhuma das variáveis antropométricas investigadas (Tabela 4). Já o tempo total semanal de tela e o de computador tiveram correlação baixa, mas significativa com massa corporal, IMC e IMC-I dos adolescentes. O nível de correlação variou muito pouco ao se controlar pela idade (Tabela 4) ou pelo fato de o adolescente ter o tempo vendo televisão controlado pelo somatório de atividade física semanal (dados não apresentados). 
Tabela 2

Estado nutricional (\%), usando o índice de massa corporal para idade (IMC-I), por sexo, segundo faixa etária dos escolares da Rede Pública Municipal de Ensino Fundamental de Niterói, Rio de Janeiro, Brasil, 2010.

\begin{tabular}{|c|c|c|c|c|c|}
\hline \multirow[t]{3}{*}{ Faixa etária (anos) } & \multicolumn{5}{|c|}{ Estado nutricional (IMC-I) } \\
\hline & \multicolumn{5}{|c|}{ Meninos } \\
\hline & $\mathrm{n}$ & $\begin{array}{c}\text { Baixo peso } \\
(Z<-2)\end{array}$ & $\begin{array}{c}\text { Normal } \\
(-2 \leq Z \leq+1)\end{array}$ & $\begin{array}{l}\text { Sobrepeso } \\
(+1<Z \leq+2)\end{array}$ & $\begin{array}{l}\text { Obesidade } \\
\qquad(Z>+2)\end{array}$ \\
\hline$<14$ & 796 & $1,5^{\mathrm{a}}(0,7 ; 2,4)$ & $67,0 *(63,7 ; 70,2)$ & $21,9 *(19,1 ; 24,8)$ & $9,6 *(7,5 ; 11,6)$ \\
\hline$\geq 14$ & 668 & $2,1(1,0 ; 3,1)$ & 81,8 * $(78,8 ; 84,7)$ & $14,0 *(11,4 ; 16,6)$ & $2,2^{a} *(1,1 ; 3,3)$ \\
\hline \multirow[t]{3}{*}{ Todos } & 1.464 & $1,8 \mathrm{a}(1,1 ; 2,5)$ & $73,7(71,5 ; 76,0)$ & $18,3(16,3 ; 20,3)$ & $6,2 \mathrm{a}(4,9 ; 7,4)$ \\
\hline & \multicolumn{5}{|c|}{ Meninas } \\
\hline & $\mathrm{n}$ & $\begin{array}{c}\text { Baixo peso } \\
(Z<-2)\end{array}$ & $\begin{array}{c}\text { Normal } \\
(-2 \leq Z \leq+1)\end{array}$ & $\begin{array}{c}\text { Sobrepeso } \\
(+1<Z \leq+2)\end{array}$ & $\begin{array}{l}\text { Obesidade } \\
(Z>+2)\end{array}$ \\
\hline$<14$ & 1.805 & $0,0 a *(-)$ & $69,9 *(67,8 ; 72,0)$ & $20,2 *(18,4 ; 22,1)$ & $9,9 *(8,5 ; 11,2)$ \\
\hline$\geq 14$ & 1.277 & $1,5 *(0,8 ; 2,2)$ & $77,5 *(75,2 ; 79,7)$ & $14,6 *(12,6 ; 16,5)$ & $6,4 a *(5,1 ; 7,8)$ \\
\hline Todos & 3.082 & $0,6 \mathrm{a}(0,3 ; 0,9)$ & $73(71,5 ; 74,6)$ & $17,9(16,5 ; 19,2)$ & 8,5 a $(7,5 ; 9,4)$ \\
\hline
\end{tabular}

* Estimativas de porcentagens significativamente (5\%) diferentes entre as duas faixas de idade.

Nota: estimativas de porcentagens de uma mesma linha com a letra "a" são significativamente (5\%) diferentes entre meninos e meninas.

Tabela 3

Tempo total semanal (horas) assistindo à televisão, jogando videogame, usando computador, o total destas atividades somadas (tela) e de realização de atividade física, por sexo, segundo faixa etária dos escolares da Rede Pública Municipal de Ensino fundamental de Niterói, Rio de Janeiro, Brasil, 2010.

\begin{tabular}{|c|c|c|c|c|c|c|c|c|c|c|}
\hline \multirow[t]{4}{*}{ Faixa etária (anos) } & \multicolumn{10}{|c|}{ Média de tempo total semanal (horas) } \\
\hline & \multicolumn{10}{|c|}{ Meninos } \\
\hline & \multicolumn{2}{|c|}{ Televisão } & \multicolumn{2}{|c|}{ Videogame } & \multicolumn{2}{|c|}{ Computador } & \multicolumn{2}{|c|}{ Tela } & \multicolumn{2}{|c|}{ Atividade física } \\
\hline & Média (EP) & IC95\% & Média (EP) & IC95\% & Média (EP) & IC95\% & Média (EP) & IC95\% & Média (EP) & IC95\% \\
\hline$<14$ & $18,0(1,5) \mathrm{a}$ & 15,$0 ; 21,0$ & $9,8(1,4) \mathrm{a}$ & 7,$0 ; 12,6$ & $10,0(1,4) \mathrm{a}$ & 7,$3 ; 12,8$ & $37,9(2,5)$ & 32,$9 ; 42,8$ & $5,4(0,9)$ & 3,$7 ; 7,2$ \\
\hline$\geq 14$ & $23,0(2,3)$ & 18,$5 ; 27,5$ & $7,9(1,4) \mathrm{a}$ & 5,$0 ; 10,8$ & $14,2(2,3)$ & 9,$6 ; 18,8$ & $45,1(3,7)$ & 37,$7 ; 52,5$ & $5,8(1,1) \mathrm{a}$ & 3,$6 ; 7,9$ \\
\hline \multirow[t]{4}{*}{ Todos } & $20,3(1,3) a$ & 17,$7 ; 22,9$ & $8,9(1,0) a$ & 7,$0 ; 10,9$ & $11,9(1,3)$ & 9,$3 ; 14,5$ & $41,2(2,2)$ & 36,$8 ; 45,5$ & $5,6(0,7) \mathrm{a}$ & 4,$6 ; 6,9$ \\
\hline & \multicolumn{10}{|c|}{ Meninas } \\
\hline & \multicolumn{2}{|c|}{ Televisão } & \multicolumn{2}{|c|}{ Videogame } & \multicolumn{2}{|c|}{ Computador } & \multicolumn{2}{|c|}{ Tela } & \multicolumn{2}{|c|}{ Atividade física } \\
\hline & Média (EP) & IC95\% & Média (EP) & IC95\% & Média (EP) & IC95\% & Média (EP) & IC95\% & Média (EP) & IC95\% \\
\hline$<14$ & $25,6(1,4) a$ & 22,$8 ; 28,4$ & $1,8(0,4) a$ & 1,$0 ; 2,7$ & $9,9(1,1)$ & 7,$8 ; 12,1$ & $37,4(1,8)$ & 33,$8 ; 40,9$ & $3,4(0,6)$ & 2,$2 ; 4,7$ \\
\hline$\geq 14$ & $26,8(1,3)$ & 23,$2 ; 30,3$ & $2,5(0,6) a$ & 1,$4 ; 3,7$ & $11,6(1,5)$ & 8,$5 ; 14,7$ & $40,8(2,4)$ & 36,$2 ; 45,5$ & $1,7(0,5) \mathrm{a}$ & 0,$6 ; 2,7$ \\
\hline Todos & $26,1(1,1) a$ & 23,$9 ; 28,2$ & $2,1(0,3) a$ & 1,$4 ; 2,8$ & $10,6(0,9)$ & 8,$9 ; 12,4$ & $38,8(1,4)$ & 36,$0 ; 41,6$ & $2,7(0,4) a$ & 1,$9 ; 3,6$ \\
\hline
\end{tabular}

EP: erro-padrão; IC95\%: intervalo de $95 \%$ de confiança.

Nota: estimativas de médias de uma mesma linha com a letra "a" são significativamente (5\%) diferentes entre meninos e meninas. 
Coeficientes de correlação Pearson simples e ajustados por idade entre o tempo total semanal assistindo à televisão, jogando videogame, usando computador e o total destas atividades somadas (tela) e as variáveis antropométricas (massa corporal, índice de massa corporal - IMC e IMC para idade IMC-I), por sexo, dos escolares da Rede Pública Municipal de Ensino Fundamental de Niterói, Rio de Janeiro, Brasil, 2010.

\begin{tabular}{|c|c|c|c|c|c|c|c|c|c|}
\hline & \multicolumn{3}{|c|}{ Total $(N=4.546)$} & \multicolumn{3}{|c|}{ Meninos $(N=1.464)$} & \multicolumn{3}{|c|}{ Meninas $(\mathrm{N}=3.082)$} \\
\hline & $\begin{array}{c}\text { Massa } \\
\text { corporal }\end{array}$ & IMC & IMC-I & $\begin{array}{c}\text { Massa } \\
\text { corporal }\end{array}$ & IMC & IMC-I & $\begin{array}{c}\text { Massa } \\
\text { corporal }\end{array}$ & IMC & IMC-I \\
\hline \multicolumn{10}{|l|}{ Televisão } \\
\hline Coeficiente de correlação Pearson simples & 0,077 & 0,086 & 0,064 & 0,053 & 0,089 & 0,047 & 0,096 & 0,062 & 0,057 \\
\hline Coeficiente de correlação Pearson ajustado pela idade & 0,063 & 0,08 & 0,069 & $-0,009$ & 0,07 & 0,063 & 0,09 & 0,057 & 0,061 \\
\hline \multicolumn{10}{|l|}{ Videogame } \\
\hline Coeficiente de correlação Pearson simples & $-0,027$ & $-0,082$ & $-0,061$ & $-0,111$ & $-0,129$ & 0,093 & 0,041 & 0,022 & 0,012 \\
\hline Coeficiente de correlação Pearson ajustado pela idade & $-0,034$ & $-0,085$ & $-0,06$ & $-0,073$ & $-0,115$ & $-0,107$ & 0,022 & 0,011 & 0,019 \\
\hline \multicolumn{10}{|l|}{ Computador } \\
\hline Coeficiente de correlação Pearson simples & 0,278 * & 0,258 * & 0,222 * & 0,430 * & 0,353 * & 0,216 * & 0,191 * & 0,229 * & 0,231 * \\
\hline Coeficiente de correlação Pearson ajustado pela idade & 0,254 * & 0,244 * & 0,238 * & 0,352 * & 0,322 * & 0,264 * & 0,188 * & 0,226 * & 0,235 * \\
\hline \multicolumn{10}{|l|}{ Tela } \\
\hline Coeficiente de correlação Pearson simples & 0,218 * & 0,191 * & $0,161 *$ & 0,239 * & 0,207 * & 0,115 * & 0,204 * & 0,197 * & 0,191 * \\
\hline Coeficiente de correlação Pearson ajustado pela idade & 0,190 * & 0,178 * & 0,176 * & 0,166 * & 0,179 * & 0,144 * & 0,193 * & 0,189 * & 0,199 * \\
\hline \multicolumn{10}{|l|}{ Atividade física } \\
\hline Coeficiente de correlação Pearson simples & $-0,002$ & $-0,007$ & 0,036 & 0,056 & 0,086 & 0,061 & $-0,044$ & $-0,016$ & 0,043 \\
\hline Coeficiente de correlação Pearson ajustado pela idade & 0,045 & 0,01 & 0,023 & 0,063 & 0,085 & 0,062 & 0,014 & 0,153 & 0,023 \\
\hline
\end{tabular}

* Valor de $p<0,05$

A prevalência de excesso de peso (sobrepeso + obesidade) associou-se significativamente com o tempo de tela (Tabela 5). A chance de apresentar excesso de peso foi 1,864 (IC95\%: 1,446; 2,404) vez maior para os escolares de todas as idades e muito maior para os meninos $(\mathrm{OR}=3,195)$ do que para as meninas $(\mathrm{OR}=1,562)$. Para os meninos, ter tempo de tela mais de $14 \mathrm{~h} /$ semana já os coloca em maior risco de apresentarem excesso de peso. Para a população total e para as meninas isso ocorre apenas com mais de $28 \mathrm{~h}$ /semana de tempo de tela.

\section{Discussão}

O presente estudo avaliou o estado nutricional e sua associação com a atividade física e o tempo realizando atividades sedentárias (assistir à televisão, jogar videogame e usar o computador) em amostra probabilística de escolares da Rede Pública Municipal de Ensino Fundamental de Niterói. Os dados indicaram alta prevalência de excesso de peso e correlação com o tempo de tela nos jovens.

A comparação entre o crescimento linear dos adolescentes de Niterói com os adolescentes de referência da curva americana, recomendada pa- ra uso internacional pela OMS, mostrou que os de Niterói apresentam, em sua totalidade, padrão semelhante ao da população norte-americana, indicando, dessa forma, que déficit de estatura não é um problema nutricional em regiões urbanas do país 1,16 . De fato, baixa estatura foi praticamente inexistente; por outro lado, constatou-se percentual alto de excesso de peso.

As meninas escolares adolescentes de $\mathrm{Ni}$ terói apresentaram prevalência de sobrepeso semelhante à dos meninos $(0,4 \%$ menor $)$, mas os valores $(\cong 18 \%$ ) foram bastante superiores ao encontrado em outro estudo em Niterói em 2003 (10,5\% em meninos e 9\% em meninas) 17. É digno de nota que os métodos usados naquele estudo diferem dos empregados no presente estudo. Entretanto, os resultados da Pesquisa Nacional de Saúde do Escolar (PeNSE), que empregou métodos semelhantes aos deste trabalho, evidenciaram maiores prevalências de escolares com sobrepeso em Porto Alegre, Rio Grande do Sul (20,1\%), seguido do Rio de Janeiro (18,3\%) 18 , com valor quase idêntico ao dos escolares adolescentes de Niterói.

Na PeNSE, em 2009, um único ano escolar (nono) foi avaliado, e 47,1\% tinham 14 anos de idade. Usando esta idade como ponto de corte da distribuição etária, os dados dos escolares de 
Prevalência de excesso de peso (sobrepeso + obesidade) segundo tempo total de tela (horas/semana), por sexo, dos escolares da Rede Pública Municipal de Ensino Fundamental de Niterói, Rio de Janeiro, Brasil, 2010.

\begin{tabular}{|c|c|c|c|c|c|c|}
\hline \multirow{2}{*}{$\begin{array}{l}\text { Tempo de tela } \\
\text { (horas/semana) }\end{array}$} & \multicolumn{2}{|c|}{ Todos $(\mathrm{N}=4.546)$} & \multicolumn{2}{|c|}{ Meninos (N = 1.464) } & \multicolumn{2}{|c|}{ Meninas ( $N=3.082)$} \\
\hline & $\%$ & OR (IC95\%) & $\%$ & OR (IC95\%) & $\%$ & OR (IC95\%) \\
\hline$<14$ & 18,5 & 1,000 & 10,9 & 1,000 & 22,0 & 1,000 \\
\hline $14-27,9$ & 17,8 & $0,955(0,717 ; 1,272)$ & 19,4 & $1,970(1,078 ; 3,601)$ & 17,1 & $0,733(0,526 ; 1,022)$ \\
\hline$\geq 28$ & 29,7 & $1,864(1,446 ; 2,404)$ & 28,1 & $3,195(1,832 ; 5,573)$ & 30,5 & $1,562(1,169 ; 2,087)$ \\
\hline
\end{tabular}

IC95\%: intervalo de $95 \%$ de confiança; OR: odds ratio.

Niterói evidenciaram que a prevalência de sobrepeso e obesidade foram maiores nos mais jovens, tanto em meninas, quanto em meninos. Não está claro se essa diferença é influenciada por questões biológicas (maturação sexual) ou socioeconômicas, merecendo investigação futura. Assim, como evidenciado para o conjunto da população adolescente brasileira, a população escolar da Rede Pública Municipal de Ensino Fundamental de Niterói enfrenta um quadro de transição de desnutrição por déficit de estatura para o aparecimento crescente de sobrepeso/obesidade.

Esses dados sugerem uma urgente necessidade de educar a comunidade escolar adolescente urbana de Niterói e, possivelmente, a de outros municípios brasileiros, quanto aos aspectos dos estilos de vida saudáveis, para prevenir o excesso de peso e seus efeitos nocivos associados 19. A fim de que o sobrepeso e a obesidade não ocorram na infância, Goldfield et al. 20 afirmam que há necessidade de desenvolver métodos mais inovadores de motivar as crianças com sobrepeso a aumentar a atividade física e reduzir o comportamento sedentário.

O tempo diário diante de uma televisão vem sendo usado como indicador de vida sedentária, particularmente em adolescentes ${ }^{3}$. De fato, essa variável é de fácil obtenção e tem lógica, do ponto de vista explicativo, ao constituir-se em hábito que poderá diminuir a prática esportiva de lazer ${ }^{8}$ e aumentar a ingestão energética ${ }^{21}$, particularmente em crianças/adolescentes. Ao comparar estudos feitos em várias regiões do Brasil, percebeu-se que a média diária de horas vendo televisão variou muito. Em 2003, na cidade de Niterói, a média foi de $4,7 \mathrm{~h} 17$. Na presente investigação, a média foi de 3,4h e variou pouco entre os dias da semana ou de final de semana. Em estudos realizados em outros estados (Alagoas e Santa Catarina), a média diária variou de $3 \mathrm{~h}$ a $4,3 \mathrm{~h}$ por dia, dependendo do estado nutricional 22,23. Os adolescentes de Niterói assistem à televi- são, em média, por tempo acima do recomendado pela Academia Americana de Pediatria (não ver televisão por $2 \mathrm{~h}$ ou mais diariamente) 24 e do proposto por Mauriello et al. 25, como forma de prevenção da obesidade, em abordagem de base populacional. Nunes et al. 26 sugerem o limite de $3 \mathrm{~h}$ por dia como ponto para classificar adolescentes com estilo de vida inadequado. $O$ valor de $4 \mathrm{~h}$ ou mais por dia de televisão já foi sugerido como ponto para definir adolescentes como consumidores excessivos de televisão 27 .

Quase a metade dos escolares de Niterói $(47,2 \%)$ tinha TV por assinatura, significando que a oferta de programação oferecida pela televisão é diversificada. Na Austrália, pesquisa constatou que os adolescentes que têm acesso a essa possibilidade são mais propensos a assistir mais do que $2 \mathrm{~h}$ de televisão por dia ${ }^{28}$. É possível que quanto maior a duração do entretenimento televisivo, maiores sejam as chances de o adolescente fazer da televisão o passatempo preferido e gastar mais tempo diante deste lazer passivo.

Recentemente, o uso do tempo de tela, substituindo o tempo assistindo à televisão, começou a ser descrito como um marcador de comportamento sedentário de crianças e adolescentes 29. Contudo, ainda não existe uma padronização sobre como calculá-lo, pois estudos diferem quanto ao entendimento do seu significado. Existem locais que consideram três componentes na análise, enquanto outros utilizam o somatório de apenas duas atividades. Por exemplo, em Portugal, Mota et al. 29 consideraram tempo de tela com apenas dois componentes: assistir à televisão e usar o computador. Já na Nova Zelândia, chamou-se de tempo de tela o somatório do tempo de televisão e de videogames 3 . No México, Lajous et al. 30 consideraram três componentes: tempo de computador, televisão e videogames, semelhante à forma empregada nos Estados Unidos 7 e Austrália 31.

O tempo de tela dos escolares do ensino fundamental público de Niterói, calculado como o 
tempo vendo televisão, usando computador e jogando videogames, não apresentou diferença entre meninos e meninas, tampouco entre faixa etária: foi relativamente alto, chegando a mais de $5 \mathrm{~h}$ diárias, e associou-se significativamente ao excesso de peso. De fato, há documentação na literatura de associação positiva e significativa entre o tempo total semanal de tela, medidas de adiposidade (dobra cutânea e IMC) e risco metabólico em crianças e adolescentes 19,30,32,33.

Atualmente, a Austrália está fazendo esforços nacionais para aumentar a atividade física entre crianças e adolescentes que estão inseridos em comportamentos baseados em tempo de tela 8 , de forma similar ao que vem ocorrendo no México, cujas intervenções preventivas de obesidade têm explorado estratégias para reduzir o tempo de tela na adolescência ${ }^{30}$. No Canadá, pesquisa menciona que intervenções de saúde pública, pautada no estilo de vida para a juventude, devem incluir um componente específico destinado a reduzir o tempo de tela 33 . Assim, essa redução, que pode ser um meio eficaz de prevenção obesidade 34 , começa a ser recomendada nacionalmente para adolescentes em alguns países, tal como Austrália, para menos de duas horas diárias 11 .

É digno de nota o baixo tempo gasto pelos escolares em atividade física, particularmente as meninas. Atualmente, recomenda-se, para a boa saúde, que escolares devam realizar, pelo menos, sessenta minutos de atividade física diária de intensidade moderada a pesada 35 . Portanto, independentemente do tempo de tela, é necessário que os escolares do Ensino Fundamental público de Niterói aumentem a quantidade de atividade física realizada.
O fato de se basear em uma amostra probabilística de escolares da rede pública de ensino fundamental de um município brasileiro é um ponto importante do presente estudo. Todavia, a obtenção do nível de atividade física de forma subjetiva pode ser considerada uma limitação desta pesquisa; ademais não se distinguiu o tipo de videogame usado pelos escolares. Esses fatores devem ser investigados em estudos futuros.

Em conclusão, as caracterizações do estado nutricional dos escolares de Niterói apresentam valores que requerem cuidados não só quanto à prevalência de sobrepeso e obesidade, mas também quanto ao tempo assistindo à televisão (e o tempo total de tela), o que os caracteriza como adolescentes com estilo de vida inadequado. Desse modo, orientações sobre o tempo de tela acima de quatro horas diárias são importantes, pois este comportamento sedentário é modificável e pode contribuir na prevenção de excesso de peso em adolescentes. No entanto, não basta, apenas, a redução do tempo de tela. Uma vez que o ato de ver televisão, jogar videogame ou usar computador é uma realidade na vida atual dos adolescentes, sugere-se que eles conciliem essas atividades com o tempo destinado à atividade física. Como forma de compensação, para cada duas horas diárias assistindo às programações televisivas, os adolescentes deveriam gastar, pelo menos, trinta minutos de atividade física de intensidade moderada a pesada, o que daria, para os escolares da Rede Pública Municipal de Ensino Fundamental de Niterói, nível de atividade física considerado como benéfico para sua saúde. 


\section{Resumen}

El presente estudio evalúo el estado nutricional, relacionado con el tiempo dedicado a ver la televisión (computado como la suma de ver la televisión, usar el ordenador y jugar videojuegos) y la práctica de actividad física, mediante un cuestionario semiestructurado, en una muestra probabilística ( $n=328$, siendo $108 \mathrm{ni}$ ños) de escolares (10 a 18 años de edad) del sexto al noveno año de la red pública de enseñanza fundamental de Niterói, Río de Janeiro, Brasil. El déficit de estatura no fue un problema en los escolares, pero un 25,7\% tenían un exceso de peso, estando un $18 \%$ con sobrepeso y un 7,7\% con obesidad. La media de tiempo semanal de televisión fue de 24,2h, siendo mayor entre las $n i$ ñas. El tiempo dedicado a ver la televisión no fue diferente entre sexos, pero los niños, independientemente de la edad, pasaban más tiempo jugando videojuegos que las niñas. Los niños dedicaban el doble de tiempo a realizar actividades físicas, en relación con las niñas de todas las edades, y el triple en la franja de 14 años de edad o más. El tiempo de televisión se asoció significativamente con el exceso de peso. Se concluye que los escolares de la red pública de enseñanza fundamental de Niterói presentan una elevada prevalencia de exceso de peso, asociado a un estilo de vida inadecuado.

Estado Nutricional; Obesidad; Adolescente

\section{Colaboradores}

M. B. Vasconcellos colaborou no planejamento da pesquisa, coletou os dados, analisou os resultados e escreveu a primeira versão do texto. L. A. Anjos planejou a pesquisa, analisou os resultados, revisou e aprovou a versão final do manuscrito. M. T. L. Vasconcellos desenhou a amostra, analisou os resultados, revisou e aprovou a versão final do manuscrito.

\section{Agradecimentos}

Os autores agradecem a colaboração de Melina da Frota Ferreira na coleta de dados e a Solange Santiago Ferreira, Coordenadora do Núcleo de Estágio da Fundação Municipal de Educação de Niterói (NEST), por autorizar a pesquisa. Luiz Antonio dos Anjos recebeu uma bolsa de produtividade em pesquisa do CNPq (processo 308489/2009-8).

\section{Referências}

1. Instituto Brasileiro de Geografia e Estatística. Pesquisa de Orçamentos Familiares 2008-2009: antropometria e estado nutricional de crianças, adolescentes e adultos do Brasil. Brasília: Instituto Brasileiro de Geografia e Estatística; 2010.

2. Mendonça CP, Anjos LA. Aspectos das práticas alimentares e da atividade física como determinantes do crescimento do sobrepeso/obesidade no Brasil. Cad Saúde Pública 2004; 20:698-709.

3. Foley LS, Maddison R, Jiang Y, Olds T, Ridley K. It's not just the television: survey analysis of sedentary behaviour in New Zealand young people. Int J Behav Nutr Phys Act 2011; 8:132.

4. United Nations Educational, Scientific and Cultural Organization. UNESCO Statistical Yearbook 1999: culture and communication statistics. Montreal: United Nations Educational, Scientific and Cultural Organization; 1999.
5. Wang Y, Liang H, Tussing L, Braunschweig C, Caballero B, Flay B. Obesity and related risk factors among low socio-economic status minority students in Chicago. Public Health Nutr 2007; 10: 927-38.

6. Ludwig DS, Gortmaker SL. Programming obesity in childhood. Lancet 2004; 364:226-7.

7. Must A, Bandini LG, Tybor DJ, Phillips SM, Naumova EM, Dietz WH. Activity, inactivity, and screen time in relation to weight and fatness over adolescence in girls. Obesity 2007; 15:1774-81.

8. Melkevik O, Torsheim T, Iannotti RJ, Wold B. Is spending time in screen-based sedentary behaviors associated with less physical activity: a cross national investigation. Int J Behav Nutr Phys Act 2010; 7:46. 
9. Oliveira TC, Silva AAM, Santos CJN, Sousa J, Conceição SIO. Atividade física e sedentarismo em escolares da rede pública e privada de ensino em São Luís. Rev Saúde Pública 2010; 44:996-1004.

10. Sichieri R, Souza RA. Estratégias para prevenção da obesidade em crianças e adolescentes. Cad Saúde Pública 2008; 24 Suppl 2:S209-23.

11. Hardy LL, Denney-Wilson E, Thrift AP, Okely AD, Baur LA. Screen time and metabolic risk factors among adolescents. Arch Pediatr Adolesc Med 2010; 164:643-9.

12. Haldane JBS. On a method of estimating frequencies. Biometrika 1945; 33:222-5.

13. Lohman TG, Roche AF, Martorell R. Anthropometric standardization reference manual. Champaign: Human Kinetics; 1988

14. De Onis M, Onyango AW, Borghi E, Siyam A, Nishida C, Siekmanna J. Development of a WHO growth reference for school-aged children and adolescents. Bull World Health Organ 2007; 85:660-7.

15. Barros MVG, Nahas MV. Medidas da atividade física: teoria e prática em diversos grupos populacionais. Londrina: Midiograf; 2003.

16. Anjos LA, Castro IRR, Engstrom EM, Azevedo AMF. Crescimento e estado nutricional em amostra probabilística de escolares no Município do Rio de Janeiro, 1999. Cad Saúde Pública 2003; 19 Suppl 1:S171-9.

17. Silva RR, Malina RM. Sobrepeso, atividade física e tempo de televisão entre adolescentes de Niterói, Rio de Janeiro, Brasil. Rev Bras Ciênc Mov 2003; 11:63-6.

18. Instituto Brasileiro de Geografia e Estatística. Pesquisa Nacional de Saúde do Escolar 2009 (PeNSE). Avaliação do estado nutricional dos escolares do 9o ano do ensino fundamental: municípios das capitais e Distrito Federal. Rio de Janeiro: Instituto Brasileiro de Geografia e Estatística; 2010.

19. Laxmaiah A, Nagalla B, Vijayaraghavan K, Nair M. Factors affecting prevalence of overweight among 12- to 17-year-old urban adolescents in Hyderabad, India. Obesity 2007; 15:1384-90.

20. Goldfield GS, Mallory R, Parker T, Cunningham T, Legg C, Lumb A, et al. Effects of open-loop feedback on physical activity and television viewing in overweight and obese children: a randomized, controlled trial. Pediatrics 2006; 118:157-66.

21. Boyland EJ, Harrold JA, Kirkham TC, Corker C, Cuddy J, Evans D, et al. Food commercials increase preference for energy-dense foods, particularly in children who watch more television. Pediatrics 2011; 128:e93-100.

22. Rivera IR, Silva MAM, Silva RDTA, Oliveira BAV Carvalho ACC. Atividade física, horas de assistência à TV e composição corporal em crianças e adolescentes. Arq Bras Cardiol 2010; 95:159-65.

23. Pelegrini A, Silva RCR, Petroski EL. Relação entre o tempo em frente à TV, gasto e consumo calórico em adolescentes com diferentes percentuais de gordura corporal. Rev Bras Cineantropom Desempenho Hum 2008; 10:81-4.
24. Committee on Public Education, American Academy of Pediatrics. American Academy of Pediatrics: children, adolescents, and television. Pediatrics 2001; 107:423-6.

25. Mauriello LM, Driskell MM, Sherman KJ, Johnson SS, Prochaska JM, Prochaska JO. Acceptability of a school-based intervention for the prevention of adolescent obesity. J Sch Nurs 2006; 22:269-77.

26. Nunes MMA, Figueiroa JN, Alves JGB. Excesso de peso, atividade física e hábitos alimentares entre adolescentes de diferentes classes econômicas em Campina Grande (PB). Rev Assoc Med Bras 2007; 53:130-4

27. Campagnolo PDB, Vitolo MR, Gama CM. Fatores associados ao hábito de assistir TV em excesso entre adolescentes. Rev Bras Med Esporte 2008; 14:197-200.

28. Hardy LL, Baur LA, Garnett SP, Crawford D, Campbell KJ, Shrewsbury VA, et al. Family and home correlates of television viewing in 12-13 year old adolescents: the Nepean Study. Int J Behav Nutr Phys Act 2006; 3:24.

29. Mota J, Ribeiro JC, Santos MP. Obese girls differ ences in neighbourhood, screen time and socioeconomic status according to level of physical activity. Health Educ Res 2009; 24:98-104.

30. Lajous M, Chavarro J, Peterson KE, HernándezPrado B, Cruz-Valdéz A, Hernández-Avila M, et al. Screen time and adiposity in adolescents in Mexico. Public Health Nutr 2009; 12:1938-45.

31. Smith BJ, Gruseit A, Hardy LL, King L, Wolfenden L, Milat A. Parental influences on child physical activity and screen viewing time: a population based study. BMC Public Health 2010; 10:593

32. Suñe FR, Dias-da-Costa JS, Olinto MTA, Pattussi MP. Prevalência e fatores associados para sobrepeso e obesidade em escolares de uma cidade no Sul do Brasil. Cad Saúde Pública 2007; 23:1361-71.

33. Mark AE, Janssen I. Relationship between screen time and metabolic syndrome in adolescents. J Public Health 2008; 30:153-60.

34. Jago R, Baranowski T, Baranowski JC, Thompson D, Greaves KA. BMI from 3-6y of age is predicted by TV viewing and physical activity, not diet. Int J Obes 2005; 29:557-64.

35. Strong WB, Malina RM, Blimkie CJ, Daniels SR, Dishman RK, Gutin B, et al. Evidence based physical activity for school-age youth. J Pediatr 2005; 146:732-7.

Recebido em 29/Fev/2012

Versão final reapresentada em 12/Nov/2012

Aprovado em 22/Nov/2012 